\title{
Vom Kritischen Bericht zur Kritischen Dokumentation am Beispiel der Digital-interaktiven Mozart-Edition
}

\author{
NORBERT DUBOWY, SALZBURG
}

Dieser Beitrag möchte auf einige ebenso bekannte wie grundsätzliche Gegenstände und Verfahren der musikalischen Editionspraxis bei historisch-kritischen Ausgaben eingehen. Gemeint ist das Verhältnis von Edition und Kritischem Bericht bzw. die Art und Weise, wie Gegenstände und Befunde, die im Kritischen Bericht dokumentiert sind, abgelegt und referenziert werden. Die digitale Edition ist dabei in der Lage, dieses Verhältnis grundlegend neu zu formulieren, sowohl was die Kategorien von Informationen und die vermittelten Inhalte im Einzelnen betrifft als auch was die Verfügbarkeit, Sichtbarkeit und somit die Nutzbarkeit der Information innerhalb der Edition angeht. Es geht dabei letztlich um das durchaus bekannte Argument, dass die digitale Edition in der Lage ist, die Transparenz zu erhöhen und zwar nicht nur auf materieller Ebene, etwa durch die Einbindung von Quellenbildern, sondern auch auf der Ebene editorischer Prozesse und Entscheidungen. ${ }^{1}$ Den Anlass für die Beschäftigung mit dieser Thematik lieferte ursprünglich ein ,formaler' Aspekt, die Gestaltung oder Formulierung des Kritischen Berichts und insbesondere die Frage der Verbalisierung und De-Verbalisierung des kritischen Apparates in der digitalen Edition. Diese Fragestellung soll jetzt erweitert und in Bezug auf die Dokumentation und Vermittlung editorischer Prozesse ergänzt werden, $d$. h. hinsichtlich des Nachweises und der Visualisierung editorischer Eingriffe und Entscheidungen, die in digitalen Editionen sichtbar gemacht werden können, wo sie in traditionellen analogen Editionen ausgeblendet werden müssen oder nur indirekt zu vermitteln sind. Es soll dabei gezeigt werden, dass sich in einer digitalen Edition nicht nur das Format, sondern der Charakter des traditionellen Kritischen Berichts so radikal verändert, dass letztlich die Bezeichnung Kritischer Bericht selbst neu überdacht werden muss.

Als Anschauungsmaterial soll im Folgenden auf die seit dem 14. Dezember 2018 freigeschaltete Digital-interaktive Mozart-Edition (DIME) Bezug genommen werden, die an der Internationalen Stiftung Mozarteum Salzburg in Zusammenarbeit mit dem Packard Humanities Institute erarbeitet wird. Die Digital-interaktive Mozart-Edition beansprucht für sich, die erste volldigitale, funktionsfähige Musikergesamtausgabe zu sein. ${ }^{2}$ Der Bestand der in der Edition verfügbaren Werke ist zum gegenwärtigen Zeitpunkt noch begrenzt, doch wird an einer kon-

1 Siehe u. a. Partick Sahle, Digitale Editionsformen. Zum Umgang mit der Überlieferung unter den Bedingungen des Medienwandels, Teil 2: Befunde, Theorie und Methodik (= Schriften des Instituts für Dokumentologie und Editorik, Bd. 8), Norderstedt 2013, S. 184.

2 Einführung und Einstieg in die Digital-interaktive Mozart-Edition bietet die Website "Projektbeschreibung", in: Digital-interaktive Mozart-Edition, <https://dme.mozarteum.at/musik/edition/> (31.05.2020). 
tinuierlichen Ergänzung gearbeitet. Zum Projekt gehört auch das Ausloten digitaler Editionsund Codierungsmöglichkeiten im Rahmen der vorgegeben technischen Parameter (wie z. B. des gewählten Datenformats) sowie das Entwickeln von Darstellungsmodi, Visualisierungsoptionen und Funktionen für die praktische Bedienung. Insofern hat die Digital-interaktive Mozart-Edition neben der reinen Edition, der Erarbeitung und Ablage eines Notentextes, auch einen Forschungsauftrag, der sich - angesichts des relativen Neulands, den die digitale Musikedition noch darstellt - mit vielen unterschiedlichen Fragen auseinandersetzen muss. Diese reichen von der Organisation der Daten und der Codierung über die Darstellung und Vermittlung der Ergebnisse bis hin zu editionsphilologischen und -technischen Fragestellungen. Es ist diese komplexe Schichtung von Aufgaben, in der die hier präsentierten Überlegungen ihren Ursprung haben.

Dies ist zwar keine Projektpräsentation, ${ }^{3}$ es ist aber sicher hilfreich, an dieser Stelle noch einige ergänzende Information zu den Grundprinzipien der Digital-interaktiven Mozart-Edition anzufügen. Die Edition bedient sich der von der Music Encoding Initiative entwickelten Auszeichnungssprache $\mathrm{MEl}$, einem auf die Codierung von Musik und speziell auf die Anforderungen einer kritischen Edition zugeschnittenen XML-Format. ${ }^{4}$ Das gesamte musikalische Werk Mozarts wird dabei Satz für Satz in MEl codiert, wobei ein spezieller Grundsatz beachtet wird: „Die Codierung ist die Edition." Damit ist ausgedrückt, dass der Datenfile vollständig sein und alle für die Edition relevanten Informationen enthalten muss, also autonom ist. Davon getrennt ist die Visualisierung der Daten, die Rückübertragung des digitalen Codes in gängige Notenschrift. Die Digital-interaktive Mozart-Edition verwendet dazu die inzwischen weitverbreitete Music Engraving Library Verovio, ${ }^{5}$ die für dieses Projekt in eine spezifische Benutzeroberfläche eingepasst wurde, die als MoVi - The Digital Mozart Score Viewer Teil des Gesamtpaketes der DIME ist. ${ }^{6} \mathrm{MoV} i$ ist gleichsam das Instrument, mit dem die digitalen Inhalte von DIME sichtbar gemacht und interaktiv ausgewählt werden können, aber MoVi selbst ist nicht die Edition. In der Masse bietet das Projekt eine digitale Aufbereitung des Notentextes der Neuen MozartAusgabe an, die zwischen 1955 und 2007 als gedruckte Gesamtausgabe entstanden ist; es handelt sich also im Prinzip um die Formatübertragung eines gedruckten analogen Notentextes, wobei die ebenfalls gedruckten Kritischen Berichte nicht digitalisiert werden. Diese retrodigitalen Editionen tragen innerhalb des Projekts die Bezeichnung "Referenztexte”, da sie auf der Neuen Mozart-Ausgabe als der autoritativen Mozart-Edition beruhen und die Funktion einer

3 Für eine ausführliche Projektbeschreibung: siehe den Beitrag von lacopo Cividini im vorliegenden Band, „Zwischen klassischer Musikphilologie und angewandter Informatik: Die Digitale Mozart-Edition (DME) der Stiftung Mozarteum Salzburg", S. 65-83.

4 Zur Music Encoding Initiative und dem von ihr entwickelten Format siehe: Music Encoding Initiative, <https:// music-encoding.org/> (31.05.2020).

5 Entwickelt von RISM-Schweiz; Einführung und Zugang über "Home”, in: Verovio, <https://www.verovio.org/index.xhtml> (31.05.2020).

6 Zugang über die Projektwebsite bzw. "MoVi - The digital Mozart score Viewer", in: Digitale Mozart-Edition, <https://dme.mozarteum.at/movi/de> (31.05.2020). 
Referenzausgabe haben. Ergänzt werden die Referenztexte durch eine Reihe von Neueditionen ausgewählter Werke, sogenannten Alternativtexten. Es sind diese Editionen, für die einige der unten dargestellten Verfahren entwickelt wurden.

Der Kritische Bericht und der edierte Text, die "Edition“ im engeren Sinn, sind bei traditionellen analogen Ausgaben zwei "optisch getrennte ${ }^{17}$ und - in den Fällen, in denen Edition und Kritischer Bericht separat gedruckt sind - oft auch physisch voneinander getrennte Komponenten. Kritische Berichte vereinen in sich im Allgemeinen die folgenden Teile: ${ }^{8}$

a. eine Benennung und Beschreibung der Quellen,

b. eine Bewertung der Quellen, und

c. den eigentlichen Apparat, unter dem in diesem Zusammenhang der Variantenapparat oder das Lesartenverzeichnis verstanden wird. ${ }^{9}$

Es ist dieser dritte Teil, der am vielgestaltigsten ist. Er ist zuvorderst - im klassischen Variantenapparat - eine Dokumentation der Lesarten, kann jedoch auch genetische Informationen enthalten und zur Beschreibung und Beobachtung textkritischer Befunde dienen. Es handelt sich bei ihm um eine oft stark formalisierte Beschreibung, die bei Abwesenheit der Quelle selbst eine virtuelle Rekonstruktion derselben ermöglichen soll und sozusagen das Material präsentiert, ${ }^{10}$ auf dem die Edition qua ediertem Text aufbaut. In einigen Fällen, aber durchaus nicht durchgängig, dient der Apparat zusätzlich zur Mitteilung oder Begründung editorischer Entscheidungen.

Auch wenn die genannten Komponenten edierter Text und Kritischer Bericht als eigenständige Einheiten wahrgenommen werden, besteht zwischen ihnen doch eine enge Beziehung. Dem Kritischen Bericht steht - in der Musikedition mehr als in der Textedition - der eine edierte Text gegenüber, der sozusagen eine Realisierung der im Apparat beschriebenen Varianten und

7 Bodo Plachta, Editionswissenschaft, Stuttgart 1997, ${ }^{32013, ~ S . ~} 112$.

8 Der Kritische Bericht ist in der Editionspraxis und dabei insbesondere im Blickwinkel der Publikationsform der kritischen Ausgabe eine Realität, wird aber im editionsphilologischen Diskurs selten als eigenständiger Gegenstand thematisiert. So ist das, was hier beschrieben wird, im gegenwärtig maßgebenden Kompendium aufgeteilt

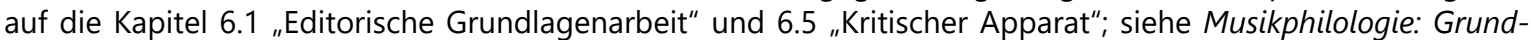
lagen, Methoden, Praxis, hrsg. von Bernhard R. Appel und Reinmar Emans (= Kompendien Musik, Bd. 3), Laaber 2017.

9 In einer anderen Definition, der hier nicht gefolgt wird, sind Kritischer Bericht und Kritischer Apparat gleichgesetzt; siehe Daniela Philippi, „Kritischer Apparat”, in: Musikphilologie: Grundlagen, Methoden, Praxis, hrsg. von Bernhard R. Appel und Reinmar Emans (= Kompendien Musik, Bd. 3), Laaber 2017, S. 237-246, hier S. 237: „Für diese quellen- und textkritischen Nachweise nutzt die Musikphilologie den Kritischen Apparat, oftmals auch als Kritischer Bericht bezeichnet."

10 Der Kritische Bericht beruht für sich bereits auf verschiedenen Arbeitsprozessen, von denen an dieser Stelle insbesondere die Kollationierung hervorgehoben werden soll. 
Möglichkeiten ist. Zwischen den beiden liegt der essentielle Arbeitsprozess der Textkonstitution, also die textkritische Herstellung des edierten Textes. ${ }^{11}$

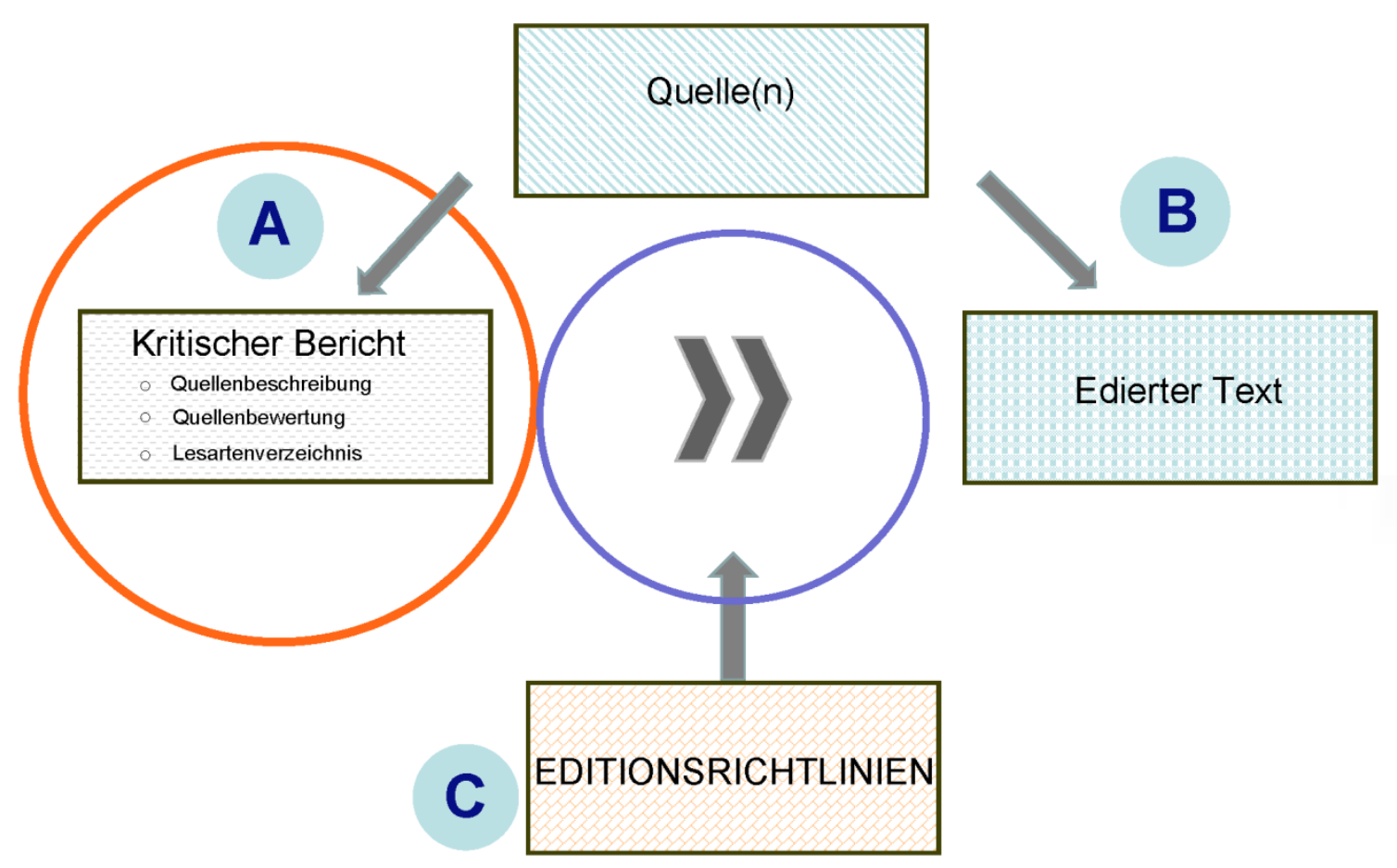

Abbildung 1: Diagramm Edierter Text - Kritischer Bericht

Das Verhältnis lässt sich modellhaft in einer einfachen Graphik veranschaulichen (Abbildung 1): Beide Komponenten haben die musikalischen Quellen zum Ausgangspunkt. Während die erste Komponente, der Kritische Bericht (A), alles festhält und beschreibt, was mit den Quellen zusammenhängt, also einen eher dokumentierenden Charakter hat, ist die zweite Komponente (B) das Ergebnis eines Prozesses, der auf den in A abgelegten Informationen aufbaut. Erkennbar wird hier auch, dass es noch einer dritten Komponente $(C)$ bedarf, die für die Steuerung dieses Prozesses verantwortlich ist. Diese dritte Komponente ist in den Editionsrichtlinien gegeben, die - meist neben einer Vielzahl organisatorischer Details - die textkritischen Grundlagen der Edition beschreiben, die kodifizierten Regeln und Konventionen benennen sowie Handlungsanweisungen für Operationen der Editoren niederlegen.

Kommen wir noch einmal zurück zum Kritischen Bericht mit seinen drei Teilen, der Quellenbeschreibung, der Quellenbewertung und dem Apparat, und fragen wir, wo und in welcher

11 Siehe die für die musikalische Editorik griffige Bestimmung in Ulrich Leisinger, „Editorische Maßnahmen: Textkonstitution," in: Musikphilologie: Grundlagen, Methoden, Praxis, hrsg. von Bernhard R. Appel und Reinmar Emans (= Kompendien Musik, Bd. 3), Laaber 2017, S. 197-227, hier S. 197. Dort als Arbeitsschritt der Deutung der in der editorischen Grundlagenarbeit erstellten Befunde. 
Weise eine digitale Edition gegebenenfalls anders vorgehen kann als eine analoge Druckausgabe.

Zu 1. Quellenbeschreibung: Eine Quellenbeschreibung folgt in der Regel einem traditionellen, von der Sache diktierten, aber im einzelnen Projekt festgelegten Raster. Hierzu zählen die Beschreibung von Fundort, Provenienz, Umfang, Format, Wasserzeichen und anderen Parametern.

Da die Beschreibung an sich schon sehr stark formalisiert ist, liegt eine Erfassung in einem strukturierten elektronischen Dokument etwa als Datenblatt oder Datenbankauszug oder generell in einem digitalen Format nahe. Im Hybrideditionsprojekt "OPERA" beispielsweise ist die Quellenbeschreibung als Textdokument im XML-Format nach den Standards der Text Encoding Initiative (TEI) abgefasst. ${ }^{12}$ Auch in der DIME sind Quellenbeschreibungen gegenwärtig noch als Textdokument in TEl angelegt, d. h. die Beschreibung steht im ,body' der XML-Datei. Besser, wenngleich im Moment noch aufwändig, ist es, die Informationen im ,header' einer XML-Datei abzulegen, der für die Aufnahme großer Mengen von Metadaten konzipiert ist. Dem Vorbild der TEl folgend, die in ihrem Schema (P5) Elemente und Attribute für die Beschreibung von handschriftlichen Quellen bereitstellt, ${ }^{13}$ hat auch die MEI mit dem letzten großen Release ihres XML-Schemas (4.0.0) Werkzeuge für die detaillierte Quellenbeschreibung entwickelt und die Beschreibungssysteme und -parameter verfeinert. ${ }^{14}$ Der Ort für eine katalogisierende Erfassung eines Werkes etwa wie in einem Werkkatalog, für eine umfassende Quellenbeschreibung oder die Quellenreferenz im Rahmen einer Edition ist prinzipiell immer der Gleiche: der DateiHeader als obligatorischer Teil eines XML-Datenfiles. Damit besteht zwischen den drei Anwendungsbereichen Werkkatalogeintrag, Quellenbeschreibung und Metadaten des edierten Texts kein grundsätzlicher, sondern nur noch ein gradueller Unterschied. In der Konsequenz bedeutet dies, dass es - sofern dies gewünscht ist - möglich ist, die MEI-Daten zur Edition des Notentexts einerseits und alle Informationen zum Werk und den Quellen andererseits direkt in ein und demselben File abzulegen. Hierbei verschwindet insbesondere die Lücke zwischen der Quellenbeschreibung (im Kritischen Bericht) und der Notenedition. Ein weiterer Vorteil der Ablage von werk- und quellenspezifischen Daten im Datei-Header ist klar: Durch die Verwendung einer vorgegebenen, definierten Struktur sind die Daten maschinenlesbar und durch den Gebrauch von Normdaten und kontrolliertem Vokabular über den lokalen Ursprungsort vernetzbar und weiterverwendbar.

12 In dieser Form etwa in Giambattista Casti und Antonio Salieri, Prima la musica poi le parole: divertimento teatrale in un atto; operetta a quattro voci, hrsg. von Thomas Betzwieser (Musik) und Adrian La Salvia (Text) (= OPERA - Spektrum des europäischen Musiktheaters in Einzeleditionen, Bd. 1), Kassel 2013. Zur Text Encoding Initiative siehe TEl: Text Encoding Initiative, <https://tei-c.org/> (31.05.2020).

13 Vgl. „P5: Guidelines for Electronic Text Encoding and Interchange. Abschnitt 10, ,Manuscript Description"“, in: TEl: Text Encoding Initiative, <https://tei-c.org/release/doc/tei-p5-doc/en/html/MS.html> (31.05.2020).

14 Kapitel 3 der Richtlinien: „3. Metadata in MEl.”, in: Music Encoding Initiative, <https://music-encoding.org/guide lines/v4/content/metadata.html> (31.05.2020), siehe insbesondere Abschnitt "3.7. Encoding Sources in MEl”. 
Zu 2. Quellenbewertung: Hier handelt es sich um eine Beurteilung oder Expertise, die sich immer am konkreten Objekt orientieren muss und sich zu einer rasterhaft strukturierten Aufbereitung und Präsentation wenig eignet. Sie wird daher wohl auch in der digitalen Edition am ehesten als fortlaufender Worttext zu konzipieren sein, der sich beispielsweise in einem eigenen TEl-Dokument oder auch im Datei-Header der Musikedition unterbringen lässt.

Zu 3. Der Kritische Apparat bzw. das Lesartenverzeichnis: Schaut man sich derartige Apparate an, so wird man erkennen, dass sie - wie zuvor schon festgestellt - viel Unterschiedliches beinhalten können. Als Beispiel mag der Apparat zum ersten Satz des Streichquartetts KV 458 von Wolfgang Amadé Mozart in der Neuen Mozart-Ausgabe dienen, der mit "Bemerkungen zu den Quellen $A$ und $B^{11}$ überschrieben ist: ${ }^{15}$ Erwartungsgemäß handelt es sich bei der Mehrzahl der Einträge um Varianten und alternative Lesarten in den beiden Quellen. Es gibt aber auch vieles, das dem Charakter nach eher nur beschreibend ist, wobei es sich nicht unbedingt um musikalisch distinktive Varianten handelt wie etwa beim ersten Eintrag, der sich auf die "Anordnung der Vorzeichen" in Quelle A bezieht. Sodann gibt es Verweise auf Erklärungen ("siehe die Bemerkung zu T. 138 ff."), in diesem Fall Mozarts Praxis der Notierung struktureller Wiederholungen, ferner die Feststellung von Vorhandenem oder Fehlendem ("ohne Augmentationspunkt"; „irrtümlich ohne 8tel-Fähnchen“), was nur bedingt als musikalische Alternative behandelt ist. Schließlich gibt es etliches an genetischer Information, etwa zu diversen Korrekturprozessen (z. B. die Anmerkung zu T. 5). In einigen wenigen Fällen kommt es auch zur Begründung editorischer Eingriffe („in NMA an T. 15/16, 16/17, angeglichen.").

Für diese heterogenen Informationen mag es in der digitalen Edition unterschiedliche Wege der Behandlung geben. Für genetische Prozesse wurde in der Frühzeit der Digital-interaktiven Mozart-Edition eine bevorzugt auf Codierung und visueller Darstellung beruhende Umsetzung versucht, diese aber mittlerweile wieder aufgegeben, ${ }^{16}$ um stattdessen auf die lokale Anmerkung mit verbalem Kommentar zurückzukommen, wie sie vor allem in der Quellenedition des ersten Satzes von KV 458 nach dem Autograph zur Anwendung kommt. ${ }^{17}$

In anderen Fällen, wie etwa der Variantendarstellung, erweist sich die rein visuelle Anzeige ohne einen zusätzlichen Wortkommentar als vollkommen ausreichend. Da die in der Digital-

15 Ludwig Finscher und Wolf-Dieter Seiffert, Wolfgang Amadeus Mozart, Neue Ausgabe sämtlicher Werke, Kritische Berichte, Serie VIII, Werkgruppe 20, Abteilung 1: Streichquartette, Bd. 2, Kassel 1992, S. 74ff; zugänglich als NMA Online, hrsg. von der Internationalen Stiftung Mozarteum, Salzburg, <https://dme.mozarteum.at/DME/nma/ nma_cont.php?vsep=176\&gen=kb\&p1 =74> (31.05.2020).

16 Noch andeutungsweise sichtbar auf dem Poster der Digital-interaktiven Mozart-Edition für die Music Encoding Conference 2017 in Tours/Frankreich; siehe Norbert Dubowy, Felix Gründer, Franz Kelnreiter et al. (Stiftung Mozarteum Salzburg in Cooperation with The Packard Humanities Institute, Los Altos, CA.), The Digital Mozart Edition as a Digital Music Edition, 2017, <https://dme.mozarteum.at/DME/objs/content/poster_tours2017.pdf> (31.05.2020).

17 Lokale Anmerkungen (Annotationen) können in MoVi über das Dashboard und die Schaltfläche Annotationen/ Annotations aktiviert werden, wobei zwischen "DME-observation" (bevorzugt für kodikologisch beschreibende Befunde) und "DME-comment" (für textkritische Befunde mit Auswirkungen auf die Interpretation) unterschieden ist. 
interaktiven Mozart-Edition realisierten Neueditionen auf dem Prinzip der Quellenedition nach einer einzigen Quelle beruhen, ist die Zahl der Varianten naturgemäß gering. Das Prinzip der Variantencodierung ist jedoch in den Referenztexten, dem digitalisierten Notentext der NMA, bei der Codierung von Ossias verwirklicht, wie sie beispielsweise in der Edition des Hornquintetts KV 407 vorkommen. ${ }^{18}$

Es wird also wohl letztlich auf eine Kombination von Visuellem und Verbalem hinauslaufen, wo sich die visuelle Inhaltsvermittlung allein in ihrer Aussagekraft als zu begrenzt erweist. Auf alle Fälle hat die digitale Edition den Vorteil beides im Sinne eines lokalen „Einblendungsapparates" innerhalb des edierten Textes anzeigen zu können. Impulse für die Behandlung genetischer Prozesse im Rahmen einer digitalen Musikedition sind vor allem vom Projekt "Beethovens Werkstatt" zu erwarten, das speziell auf die Verbindung genetischer Textkritik und digitaler Musikedition ausgerichtet ist. ${ }^{19}$

Im Folgenden soll auf den Komplex der eigentlichen editorischen Einrichtung eines musikalischen Textes eingegangen werden. Dieser Bereich wird, wie bereits erwähnt, durch allgemeine und spezifische Regeln gesteuert, die in den Editionsrichtlinien oder vergleichbaren Dokumenten niedergelegt sind. Im Prinzip sind es hier zwei unterschiedliche Operationen, die zum Tragen kommen: Zum einen geht es um die formalen Aktualisierungen des Notentexts (1). In einem zweiten Schritt geht es um die editorischen Eingriffe und Entscheidungen im eigentlichen Sinn (2).

$\mathrm{Zu}$ 1. Ein großer Teil der zu leistenden Arbeit bei der Erstellung des edierten Notentexts ist zunächst formaler Natur. Hier handelt es sich um Operationen, die dem Bereich der Normalisierung bzw. Regulierung nach zuvor festgelegten, projektspezifischen Kriterien sowie der Standardisierung nach allgemein verbindlichen Regularien, gegebenenfalls auch der Modernisierung zuzuordnen sind. Während letztere sich auf die Anpassung an allgemeine Regeln und Konventionen des Notensatzes beziehen, wird es bei den Normalisierungen eher um die Anwendung spezifischer Konventionen gehen, die nur für das Projekt oder eine bestimmte Edition gelten oder die sich aus Gegebenheiten der zu edierenden Quelle ableiten lassen.

Die Maßnahmen, die zunächst nur einen gleichsam technischen Arbeitsschritt umschreiben, aber nichtdestotrotz einen Eingriff in den ursprünglichen Text der Quelle darstellen, haben nur das Ziel, einen kohärenten, an die Gepflogenheiten der Zeit und der Musikpraxis angepass-

18 Wolfgang Amadé Mozart, Quintett in Es für Horn, Violine, zwei Violen und Bass, KV 407, zweiter und dritter Satz, siehe in: MoVi - The digital Mozart score Viewer, <https://dme.mozarteum.at/movi/navigator/407/002/01>; und <https://dme.mozarteum.at/movi/navigator/407/003/01> (31.05.2020).

19 „Beethovens Werkstatt: Genetische Textkritik und Digitale Musikedition” ist ein Projekt der Musikhochschule Detmold/Universität Paderborn und des Beethoven-Hauses Bonn, gefördert durch die Akademie der Wissenschaften und der Literatur | Mainz; Informationen auf Beethovens Werkstatt. Genetische Textkritik und digitale Musikedition, <https://beethovens-werkstatt.de/> (31.05.2020). 
ten Text vorzulegen. Dies umfasst alle strikt formalen Angleichungen wie beispielsweise die einheitliche Schreibweise und Positionierung von Dynamik und Spielanweisungen, für die es in den meisten Fällen unerheblich sein dürfte, wie sie geschrieben sind. Mit der Anwendung einer standardisierten Partituranordnung oder der Anpassung an moderne Akzidenziensetzung stößt man unter Umständen jedoch bereits in Grenzbereiche vor, wo sich die formale Aktualisierung der Interpretation des originalen Textes annähert, weshalb hier besondere Sensibilität geboten ist. Zu den Regulierungen zählen auch Operationen wie die Auflösung von Notationsabbreviaturen ( $z$.B. Colla Parte, Ottava) und das Einfügen von nicht vollständig ausnotierten Abschnitten und Wiederholungen (z. B. interne Reprisen), also das Auflösen aller Arten von Kopieranweisungen.

Vieles von dem, was bisher erwähnt wurde, ist in herkömmlichen Editionen oft durch Regeln abgedeckt, die explizit oder implizit als "stillschweigend" deklariert sind, oder die als generische Handlungsanweisung für die Einrichtung des Notensatzes in den Editionsrichtlinien dokumentiert sind. In den meisten Fällen sind die Ergebnisse dem unmittelbaren Nachvollzug im Notenbild jedoch entzogen und im Einzelnen nicht nachgewiesen. ${ }^{20}$ Es besteht also ein gewisses Dokumentationsdefizit, dem in digitalen Editionen, die über ein entsprechendes Instrumentarium verfügen, zu einem großen Teil abgeholfen werden kann. Das in MEl verfügbare Instrumentarium an Auszeichnungen ermöglicht die Dokumentation von Normalisierungen im digitalen Code und damit deren potentielle Visualisierung, alternativ als originale oder als regulierte Lesart. ${ }^{21}$

Zu 2. Geht es im ersten Schritt um die formale Kohärenz des Notentextes, so steht bei dem an dieser Stelle summarisch als „editorische Eingriffe" zusammengefassten Schritt die musikalische und damit oft auch ästhetische Sinnhaftigkeit im Zentrum. Sie stellen in vielen Fällen eine gewisse Grauzone dar, die nur zum Teil dokumentarisch abgedeckt ist. Doch bevor wir zu den Dokumentationsdefiziten kommen, ist auch hier zunächst eine Unterscheidung angebracht.

Nach den Erfahrungen in der Digital-interaktiven Mozart-Edition lassen sich die editorischen Eingriffe überwiegend auf zwei Operationen reduzieren: Zum einen geht es um die Ergänzung, also das Hinzufügen von Elementen, wo in der ursprünglichen Quelle nichts steht, z. B. Dynamik oder Artikulation (a). Dabei mögen die Veranlassung oder die Art der Ergänzung, z. B. freie Ergänzung, Analogieergänzung oder Ergänzung wegen Textverlust, an dieser Stelle außer Acht gelassen werden. Die zweite Operation ist die der Interpretation der Lesart einer Quelle und damit unter Umständen die Veränderung von etwas bereits Gegebenem, etwa die Verschiebung

20 Erwähnt sei, dass beispielsweise im Projekt „OPERA” gemäß der General Editorial Policy abgeleitete Stimmen einer Partitur graphisch markiert sind: „In the Music Edition, doubling indications (e.g. unisono, colla parte, col basso) are generally written out, and the passages concerned are marked by ticks pointing upwards $[$ ] or downward 1 to the reference stave." Siehe Casti/Salieri, Prima la musica poi le parole.

21 Siehe dazu insbesondere die digitale Edition des ersten Satzes von KV 458 nach dem Autograph innerhalb der Digital-interaktiven Mozart-Edition; Aufruf in MoVi über das Dashboard und die Schaltfläche Regulierungen/Regularizations. Die Auflösung von Notationsabbreviaturen wurde auch im Projekt "Freischütz digital" verwirklicht; siehe Edition Freischütz Digital, <https://edition.freischuetz-digital.de/> (31.05.2020). 
des Anfangs- oder Endpunktes eines undeutlich gezogenen Bogens (b). Auch hier sind wieder verschiedene Anlässe für den editorischen Eingriff denkbar, sei es die Anpassung einer Stimme an den musikalischen Kontext oder an eine diachrone Analogie (Parallelstelle), sei es die Korrektur eines offensichtlichen Fehlers oder die editorische Entscheidung im Falle einer unklaren Lesart. Diese Veränderung im Sinne einer Neuinterpretation ist im Projekt als Konjektur definiert, was sich mit einigen Definitionen dieses Begriffs der Textkritik vereinbaren lässt..22

(a) Für die editorische Ergänzung bedient sich auch die traditionelle analoge Musikedition eines Mittels des Nachweises und zwar in Form der diakritischen Auszeichnung, nicht im Kritischen Bericht, sondern im edierten Notentext selbst: Der Begriff "Auszeichnung", also "Markup", besagt schon, dass es sich im Prinzip um eine Form der in diesem Fall typographischen Codierung handelt. Sie belegt grundsätzlich das Bemühen, über die editorischen Eingriffe Rechenschaft abzulegen. Verschiedene Editionsprojekte modulieren ein überschaubares Repertoire an typographischen Auszeichnungsmöglichkeiten: runde, eckige, spitze Klammern, gestrichelte oder gepunktete Linien, kursiv und gerade Buchstaben, große oder kleine Zeichen. Von einem praktischen Standpunkt aus betrachtet sind nicht alle Lösungen sinnfällig und nicht immer gut nachzuvollziehen.

Selbstverständlich hat diese Form der typographischen Auszeichnung ihr Äquivalent im digitalen Markup, wo Ergänzungen leicht durch ein bestimmtes Element im Code markiert und durch eine entsprechende Visualisierung kenntlich gemacht werden können. In den Referenztexten, den retrodigitalen Texten auf Grundlage der Neuen Mozart-Ausgabe, die im Projekt der Digital-interaktiven Mozart-Edition angeboten werden, sind denn auch alle identifizierbaren, d. h. typographisch belegten Ergänzungen der Bandherausgeber, die sich aus der genannten Palette von Auszeichnungsmöglichkeiten bedienen, einheitlich durch Einfärben oder farbiges Hinterlegen des Zeichens kenntlich gemacht.

(b) Nun zum zweiten Fall, dem interpretierenden, korrigierenden und verändernden Eingriff des Editors: Dies ist die eigentliche Grauzone des editorischen Prozesses. Es mag zwar sein, dass wissenschaftliche bzw. historisch-kritische Notenausgaben das Ziel verfolgen, "alle editorischen Entscheidungen - seien sie nun quellenbezogener, stofflicher oder inhaltlicher Art - nachvollziehbar"23 im Kritischen Apparat aufzuzeigen. Vielfach ist es jedoch so, dass Eingriffe dieser Art weder im Notentext typographisch ausgezeichnet noch im Kritischen Bericht konsequent beschrieben werden.

Es besteht also auch in diesem Fall ein gewisses Dokumentationsdefizit und zwar auf beiden Seiten. Auf Seiten des Kritischen Berichts deshalb, weil in traditionellen Editionen - mit gewissen

22 Gemeint ist die Konjektur als Verbesserung oder Richtigstellung einer Textstelle ohne Zuhilfenahme anderer Quellen, was im Falle einer Quellenedition, die auf einer einzigen Quelle basiert ohnehin gegeben ist. Vgl. Kai Bremer und Uwe Wirth, "Konjektur und Krux. Methodentheoretische und begriffsgeschichtliche Vorüberlegungen", in: Konjektur und Krux. Zur Methodenpolitik der Philologie, hrsg. von Anne Bohnenkamp et al., Göttingen 2010, S. 13-33, hier S. 14. Siehe auch Leisinger, "Editorische Maßnahmen”, S. 205.

23 Philippi, „Kritischer Apparat”, S. 237. 
Ausnahmen ${ }^{24}$ - Entscheidungen im Kritischen Bericht nicht aktiv begründet werden. Im edierten Text ist die Lesart niedergelegt, für die sich der oder die Editorln - unter Umständen auch gegen die Quelle - entschieden hat, im Kritischen Bericht steht gegebenenfalls die andere Lesart, die für den edierten Text verworfen wurde. Das ist sozusagen ein passives Verhältnis des Kritischen Berichts zur Edition; die Art der Entscheidung erschließt sich erst aus dem Vergleich von ediertem Text und Kritischem Bericht, die Begründung aber bleibt offen bzw. ist der Kompetenz des Editors anvertraut. Als Beispiel mag die Edition der Ouvertüre zu Leonore („Leonore I", 1807) von Ludwig van Beethoven dienen. Im Kritischen Bericht ist ein musikalisches Zeichen in T. 8 als "unklar" deklariert: ${ }^{25}$ Dass dies im Lesartenverzeichnis geschieht, ist sinnvoll, da es sich im konkreten Fall um potentiell zwei verschiedene Lesarten - Decrescendo oder Akzent auf der Note - handeln kann. Wie die editorische Entscheidung letztlich ausfällt, ist an dieser Stelle jedoch nicht mitgeteilt. Sie erschließt sich im Faktischen, sobald man den edierten Text selbst konsultiert, wo sich die Herausgeberinnen für die Lesart "Decrescendo" entschieden haben.

In der digitalen Edition gibt es wiederum Wege, dem Dokumentationsdefizit, das wir konstatiert haben, zu begegnen. Die Digital-interaktive Mozart-Edition hat in den eigenen Neueditionen ein Verfahren implementiert, mit dem editorische Entscheidungen ein Stück weit transparenter gemacht werden können. Das Verfahren soll hier an einem Beispiel aus dem „Jagdquartett" KV 458, erster Satz, von Wolfgang Amadé Mozart kurz vorgestellt werden: In T. 136 der autographen Partitur geht der Bogen in der ersten Violine eindeutig zur ersten Note des Folgetaktes (Abbildung 2). ${ }^{26}$ In den übrigen Stimmen aber beschränkt sich der Bogen - bei im Prinzip gleichartiger Bewegung - auf die Noten vier bis sechs von T. 136. Man wird sicher geneigt sein, die erste Violine an die übrigen Stimmen anzupassen. Das hat die Neue MozartAusgabe auch getan und in diesem Fall ihr Vorgehen sogar begründet, indem sie im Kritischen Bericht zunächst den Quellenbefund („Bogen bis zur 1. Note T. 137 gezogen“) und schließlich die editorische Maßnahme mitteilt („in NMA an die übrigen Stimmen angeglichen“). ${ }^{27}$

24 Genannt sei hier die Kritische Ausgabe der Werke von Richard Strauss, in welcher im Kritischen Bericht für jedes Einzelwerk vor dem Verzeichnis der Lesarten die Editorischen Eingriffe in die Hauptquelle aufgelistet sind. Siehe als Beispiel Andreas Pernpeintner, "Kritischer Bericht", in: Richard Strauss: Lieder mit Klavierbegleitung op. 10 bis op. 29, hrsg. von Andreas Pernpeintner (= Richard Strauss Werke. Kritische Ausgabe, II/2), <richard-strauss-aus gabe.de/b40106/kb> Version 2016, (31.05.2020), Abschnitt V „Editorische Eingriffe und Lesarten“. Das Verfahren, das festlegt, welche Eingriffe dokumentiert werden, ist ebd. in Abschnitt IV „Editionsweise und Gestaltung des Notentexts" beschrieben.

Nach Ausweis der Editionsrichtlinien sind auch in der Brahms-Gesamtausgabe Eingriffe des Herausgebers in den Notentext der Hauptquelle im Kritischen Bericht zu belegen. Siehe „Johannes Brahms Gesamtausgabe, Editionsrichtlinien (Stand 1997), Kapitel 7.6.2 Editionsbericht", in: Editionsrichtlinien Musik, im Auftrag der Fachgruppe Freie Forschungsinstitute in der Gesellschaft für Musikforschung hrsg. von Bernhard R. Appel und Joachim Veit, Kassel 2000, S. 58.

25 Ludwig van Beethoven, Werke, Abteilung IX, Bd. 1: Ouvertüren zur Oper Leonore, hrsg. von Helga Lühning und Christine Siegert, München 2017, S. 181 (Lesarten) bzw. 97 (Edition).

26 Partitur GB-Lbl Add. Ms. 37763, ff. 23r-33v: 25r, <https://www.bl.uk/manuscripts/Viewer.aspx?ref=add_ms_37763 f025r> (31.05.2020).

27 Finscher/Seiffert, KritischeBerichte, S. 78, <https://dme.mozarteum.at/DME/nma/nma_cont.php?vsep=176\&gen= kb\&p1=78> (31.05.2020). 


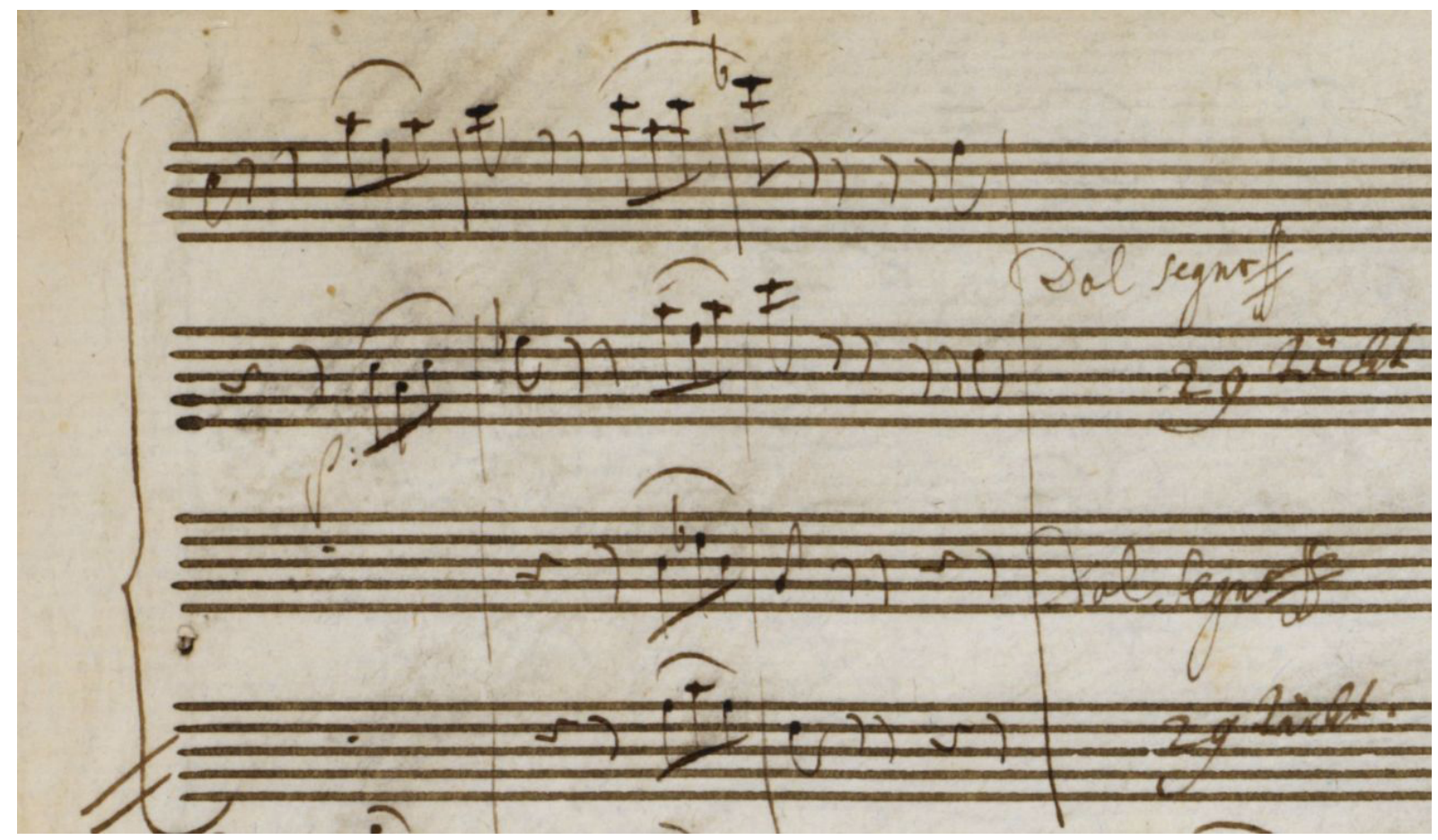

Abbildung 2: W.A. Mozart, KV 458/01, Autograph GB-Lbl, T. 135-137.

In einer digitalen Neuedition von KV 458/01 nach dem Autograph könnte der Sachverhalt hier aus der Perspektive der Visualisierung - folgendermaßen ausgedrückt sein: ${ }^{28}$ Zunächst lässt sich in MoVi - The Digital Mozart Score Viewer anzeigen, an welchen Stellen die Editoren korrigierend eingegriffen haben, indem im Dashboard über die Schaltfläche "Editorische Eingriffe" die Auswahl „Konjekturen” und schließlich "Original” bzw. nochmals „Konjektur" aufgerufen werden. Ausgehend von der emendierten Lesart wird nach Aktivierung des Kästchens "Show corresponding elements" und einem Klick auf das gewünschte Notationselement - in diesem Fall den Bogen von Violine I in T. 136 - ein Auswahlfenster eingeblendet, das nichts weiter als eine oder mehrere XML-IDs ${ }^{29}$ enthält. Der Klick auf die ID wiederum bewirkt, dass ein korrespondierendes Element farblich hinterlegt wird, das für die Korrektur als Vorlage gedient hat (Abbildung 3). Im vorliegenden Fall stellt sich dabei heraus, dass für die Verkürzung des Bogens in der Violine I - anders als in der NMA - nicht die Bögen in den anderen Stimmen als Vorbild gedient haben, sondern der Bogen in derselben Stimme einen Takt zuvor. Dies hängt damit zusammen, dass hier festgelegt wurde, analogen Ereignissen in derselben Stimme Vorrang vor parallelen Ereignissen in anderen Stimmen zu geben. Diese Regel ist selbstverständlich nicht universell, sondern muss in den Editionsrichtlinien definiert werden.

28 Siehe "MoVi - The digital Mozart score Viewer", in: Digitale Mozart-Edition, <https://dme.mozarteum.at/movi/ navigator/458/001/01 > (31.05.2020). Angezeigt wird zunächst der Referenztext; aus dem Menü „Wählen Sie eine andere Repräsentation dieses Werkes aus:" in der Übersicht ist die "Edition des Autographs" auszuwählen.

29 Die XML-ID ist der unverwechselbare Buchstaben- oder Zahlencode, der für jedes Objekt vergeben werden kann. 


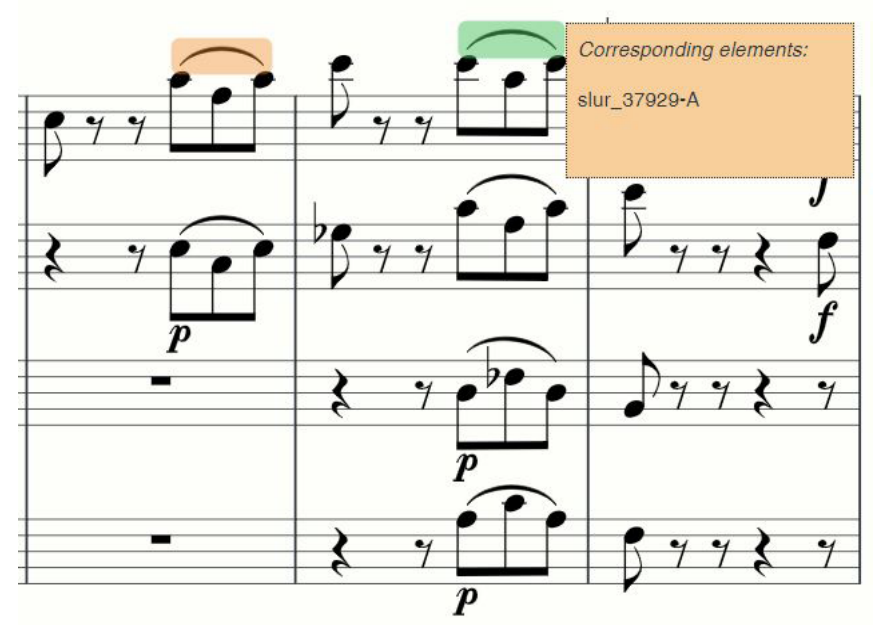

Abbildung 3: W.A. Mozart, KV 458/01, Digital-interaktive Mozart-Edition, T. 135-137.

An diesem Verfahren wird mehreres deutlich: 1. Das Dokumentationsdefizit ist aufgehoben; die editorische Angleichung selbst und die Vorlage oder Begründung, nach der die Angleichung erfolgt, werden angezeigt. Damit ist ein Maximum an Transparenz erzielt. 2. Die Dokumentation erfolgt unmittelbar im Notentext. 3. Aus dem passiven Verhältnis von lediglich beschreibendem Kritischen Apparat und ediertem Text wird eine aktive Begründung, die niederlegt, weshalb der Editor eine bestimmte Entscheidung getroffen hat. Die Edition kann sagen: „Ich habe mich entschieden, weil ...", womit praktisch der Informationsgehalt potenziert wird. 4. Der Sachverhalt der editorischen Angleichung wird grundsätzlich non-verbal vermittelt und ist prinzipiell sogar maschinenlesbar. Die Fasslichkeit kann noch durch die Integration von Quellenbildern erhöht werden, wenn man diese technisch mit einbinden möchte.

Zusammenfassend soll nun noch einmal auf generelle Aspekte eingegangen werden:

1. Unter Berücksichtigung der beschriebenen digitalen Verfahren ist es möglich, das eingangs vorgestellte Diagramm zu revidieren (Abbildung 4), wobei sich zeigt, dass sich die Linie der Dokumentation weiter in den Bereich der editorischen Operation verschiebt. Der Anteil dessen, was dokumentiert ist, umfasst nicht mehr nur die Quellenbeschreibung, -bewertung und den Variantenapparat, sondern auch weite Teile der Textkonstitution, da sowohl Teile der formalen Einrichtung des Notensatzes erfasst sind als auch Bereiche der editorischen Einrichtung einen höheren Grad an Dokumentation erhalten. 


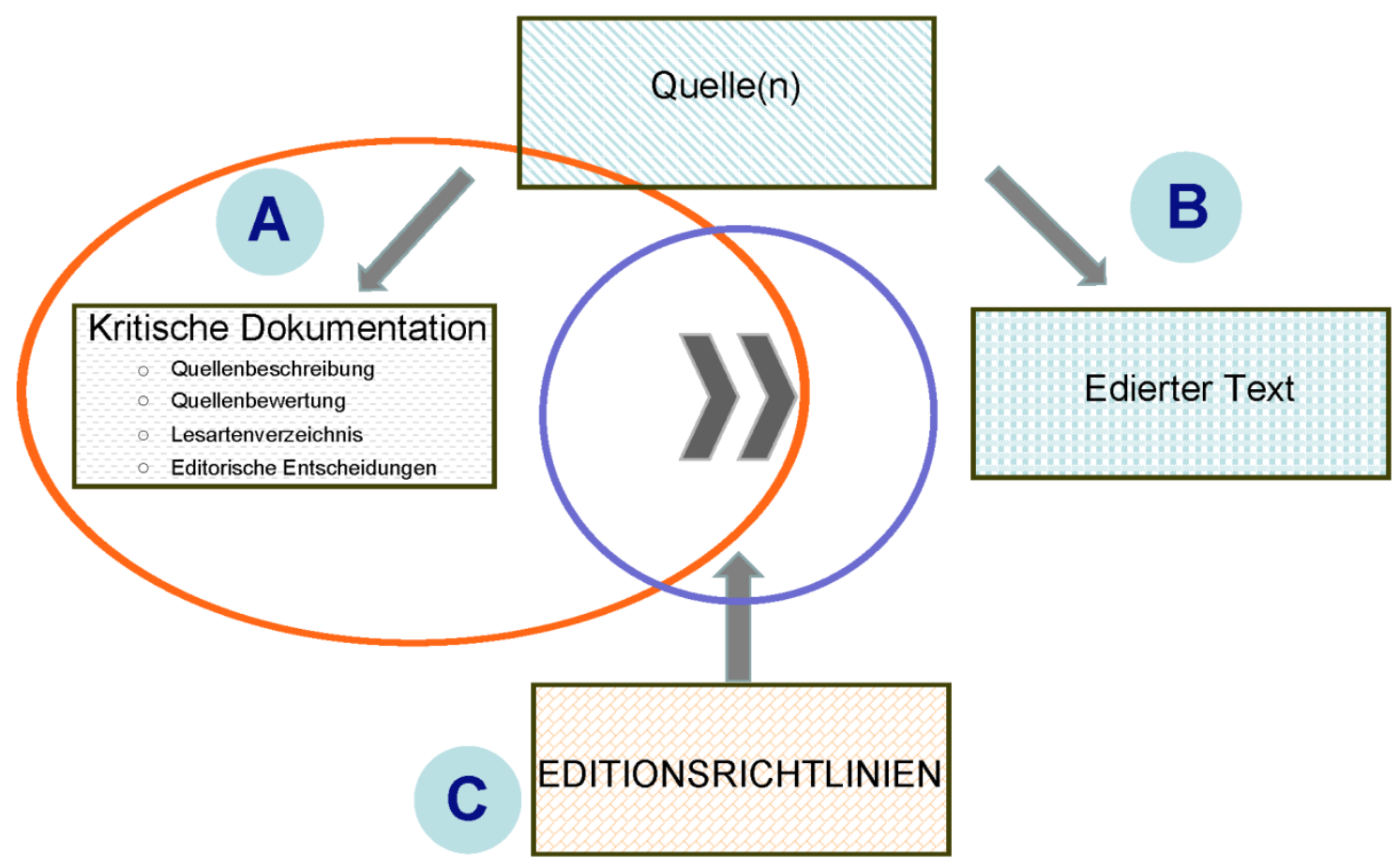

Abbildung 4: Diagramm Edierter Text - Kritische Dokumentation

2. In der digitalen Edition verschiebt sich auch die editorische Aufgabe und letztlich das, was als wissenschaftliche Leistung vorgelegt und überprüfbar wird. Damit erfüllt sich das, was Anne Baillot und Markus Schnöpf über den editorischen Prozess geschrieben haben:

„Der Editor liest die Handschrift, bereitet eine Rohtranskription vor, markiert Unsicherheiten, kommentarbedürftige Stellen, Emendationen. Dieser Arbeitsschritt hatte früher nur in den persönlichen Archiven der Editoren bzw. der Editionsvorhaben ein Dasein. Er existierte nicht als eigene wissenschaftliche, einsehbare Leistung. Dies alles umfasst aber heute die XML-Datei: sie ist das Archiv, das zum Aufbewahren dient, sie ist das, was bleibt, das, wonach man die eigentliche wissenschaftliche Leistung über die Zeiten hinweg einschätzen kann. ${ }^{130}$

Dies bestätigt auch das in der Digital-interaktiven Mozart-Edition zugrunde gelegte Axiom "Die Codierung ist die Edition", da in ihr alle editorischen und wissenschaftlichen Erkenntnisse gespeichert sind.

3. Das, was in der digitalen Edition als Dokumentation gelten kann, ist im Charakter polymorph: Es umfasst Elemente unterschiedlichster Formate, traditionellen Worttext (wie in einer Quellenbewertung), strukturierte Daten (wie in einer Quellenbeschreibung), einfaches und

30 Anne Baillot und Markus Schnöpf, "Von wissenschaftlichen Editionen als interoperablen Projekten, oder: Was können eigentlich digitale Editionen?", in: Digital Humanities: Praktiken der Digitalisierung, der Dissemination und der Selbstreflexivität, hrsg. von Wolfgang Schmale (= Historische Mitteilungen der Ranke-Gesellschaft, Beihefte Bd. 91), Stuttgart 2015, 139-156, hier S. 145-146. Hervorhebung im Original. 
komplexes Markup mit potentieller Varianz, die sowohl in Gestalt von XML-Code als auch in visualisierter Form greifbar sein kann, und letztlich auch digitale Bilder (z. B. Quellendigitalisate).

4. Da dieser Komplex so unterschiedliche Formate enthält, Textelemente, Bildelemente, mit Auszeichnungssprache geschriebenen Code, Sachverhalte, die als logische Verknüpfungen und Beziehungen existieren, sind die Grenzen fluide und nicht fest umrissen: Es ist ein teils immaterielles, virtuelles Konglomerat mit konkreten, reellen Zellen oder Kernen, das aber anders als ein Kritischer Bericht nicht zwischen zwei Buchdeckel passt.

5. Dieses Konglomerat oder "Gebilde' hätte den Begriff "Kritischer Bericht" wohl verdient, weil es nicht nur quellenspezifische und philologische Sachverhalte beschreibt, sondern auch über den Prozess der Edition Rechenschaft gibt. Aber dieser Begriff ist schon besetzt, weshalb es sinnvoll ist, dieses Gebilde fürderhin - so wie das in der Digital-interaktiven Mozart-Edition schon geschieht - die "Kritische Dokumentation" zu nennen.

Wie sich der Kritische Bericht bzw. die Kritische Dokumentation in Zukunft entwickeln werden, ist nicht vorauszusehen. Was hier versucht wurde zu beschreiben, ist die in Teilen bereits Realität gewordene Vision einer Durchdringung von Edition und Dokumentation, in der anstelle einer "leserfeindlichen" Verbalisierung eines die Notation betreffenden Sachverhaltes im Kritischen Bericht Informationen direkt im Notentext angezeigt und interaktiv, durch Filtern sowie durch Ein- oder Ausblenden nach Belieben abgerufen werden können. Dabei wird dem digitalen Medium entsprechend eine eher visuelle denn verbale Informationsvermittlung favorisiert. Auf der anderen Seite kann es sein, dass gerade die Tatsache, dass digitale Dokumente keine Platzprobleme haben und nicht auf Druckseiten Rücksicht nehmen müssen, dazu anregt, stärker als bisher zu kommentieren, zu erklären und zu vermitteln. In diesem Sinn könnte Johannes Keppers Feststellung verstanden werden, dass der "Charakter Kritischer Berichte [...] sich [...] von der Feststellung und Mitteilung der ,richtigen' Lesart hin zu einer diskursiven Betrachtung des Problems wandeln" ${ }^{\prime 1}$ werde. Wohin die Reise gehen wird, ist noch nicht zu sagen. Dazu bedarf es größerer Erfahrung mit digitaler Musikedition, die aber erst zu gewinnen ist, wenn sich mehr Projekte als bisher finden, die sich darauf einlassen, volldigital zu edieren.

Zitation: Norbert Dubowy, „Vom Kritischen Bericht zur Kritischen Dokumentation am Beispiel der Digital-interaktiven Mozart-Edition", in: Freie Beiträge zur Jahrestagung der Gesellschaft für Musikforschung 2019, hrsg. von Nina Jaeschke und Rebecca Grotjahn (= Musikwissenschaft: Aktuelle Perspektiven. Bericht über die Jahrestagung der Gesellschaft für Musikforschung 2019 in Paderborn und Detmold, Bd. 1), Detmold 2020, S. 94-108, DOI: 10.25366/2020.53.

31 Johannes Kepper, Musikedition im Zeichen neuer Medien - Historische Entwicklung und gegenwärtige Perspektiven musikalischer Gesamtausgaben, Norderstedt 2011, S. 197. 


\section{Abstract}

A digital music edition that follows the principles implemented in the fully-digital, MEl-coded Digital Interactive Mozart Edition, pursued by the Mozarteum Foundation and the Packard Humanities Institute, has many advantages over conventional analog editions. One advantage is greater transparency, which is achieved not only at the level of the material, e. g. the inclusion of digital images of the sources, but above all by making editorial processes and decisions visible in the edition itself. In the digital edition, the Critical Report, a defining component of any critical edition and often physically separate from the edited musical text, becomes part of the overall digital code. The philological findings and editorial processes reported encompass the entire range of forms of expression, from verbal comments and annotations to pure code and non-verbal, largely visual communication strategies. Therefore, the format of the traditional printed Critical Report, which is mainly made up of text and tables, dissolves and is replaced by an immaterial, non-delimitable field of data, information, references and media for which the term Critical Documentation is more appropriate.

\section{Kurzvita}

Norbert Dubowy arbeitete nach Studium und Promotion an zahlreichen Universitäten und Forschungseinrichtungen in Europa und den Vereinigten Staaten. Seit 2014 betreut er als Cheflektor an der Internationalen Stiftung Mozarteum in Salzburg die Digital-interaktive MozartEdition (DIME). Zu seinen Forschungsinteressen zählen die Musik des 17. und 18. Jahrhunderts, Librettologie und Editionsphilologie. 


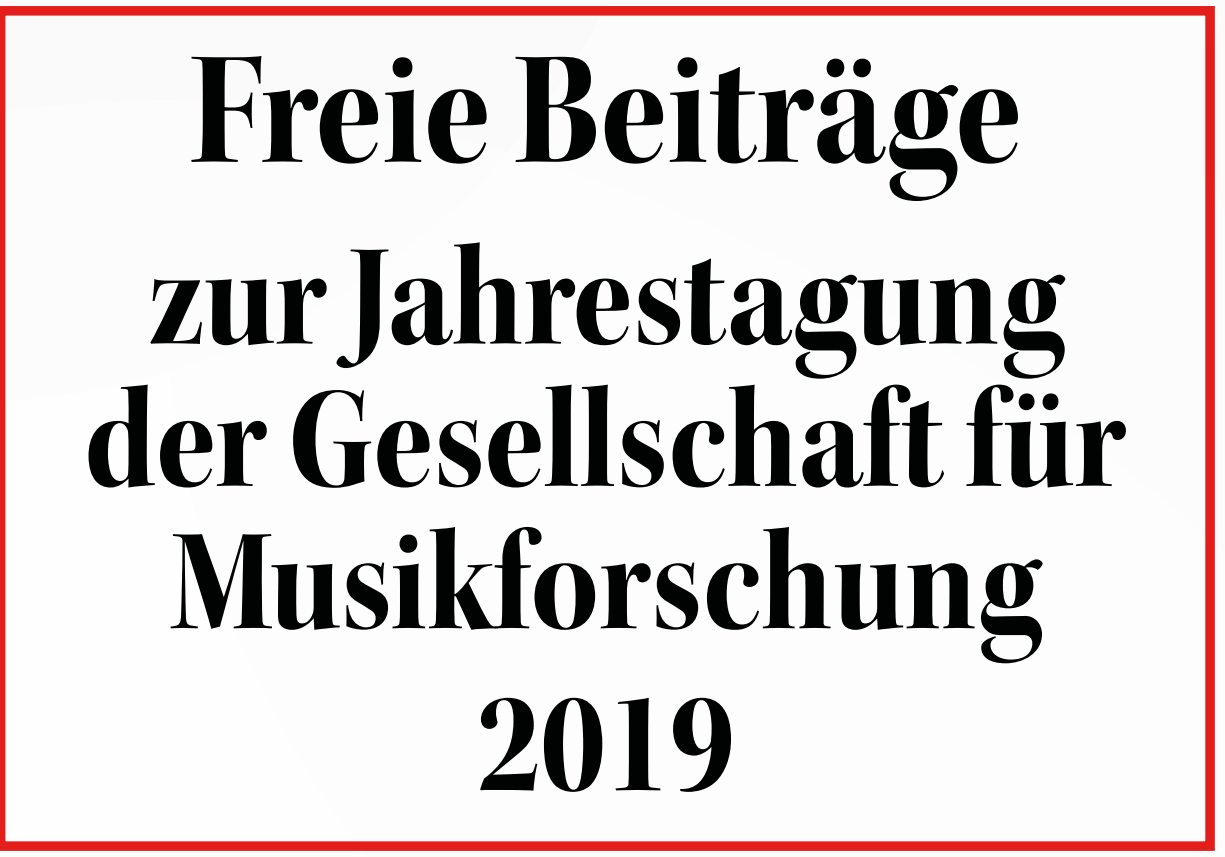

Herausgegeben von Nina Jaeschke und Rebecca Grotjahn

Musikwissenschaft: Aktuelle Perspektiven 1 
Freie Beiträge 


\section{Musikwissenschaft: Aktuelle Perspektiven}

Bericht über die Jahrestagung der Gesellschaft für Musikforschung 2019 in Paderborn und Detmold

Herausgegeben von Rebecca Grotjahn und Nina Jaeschke

Band 1 


\section{Freie Beiträge}

\section{zur Jahrestagung der Gesellschaft für Musikforschung 2019}

Herausgegeben von Nina Jaeschke und Rebecca Grotjahn

Detmold: Musikwissenschaftliches Seminar der Universität Paderborn und der Hochschule für Musik Detmold 2020 
DOI: $10.25366 / 2020.42$

Online-Version verfügbar unter der Lizenz: Urheberrecht 1.0, $<$ https://rightsstatements.org/page/InC/1.0/?language=de>

Bibliografische Information der Deutschen Nationalbibliothek

Die Deutsche Nationalbibliothek verzeichnet diese Publikation in der Deutschen Nationalbibliografie; detaillierte bibliografische Daten sind im Internet über http://dnb.d-nb.de abrufbar.

\section{Impressum}

Redaktion: Nina Jaeschke, Rebecca Grotjahn und Jonas Spieker Satz: Nina Jaeschke

(C) Musikwissenschaftliches Seminar der Universität Paderborn und der Hochschule für Musik Detmold 2020 


\section{INHALT}

Vorwort $\quad$ IX

Komponieren für das Radio: Akteure, Diskurse, Praktiken $\quad 1$

Musikwissenschaft - Feminismus - Kritik: Ein Generationenaustausch 6

\section{Stefan Alschner}

Der Wagner-Sänger Joseph Aloys Tichatschek - Vom Nachlass zum Netzwerk

\section{Alenka Barber-Kersovan}

Songs for the Goddess. Das popmusikalische Neo-Matriarchat zwischen Ethno-Beat,

erfundenen Traditionen und kommerzieller Vermarktung

Elias Berner, Julia Jaklin, Peter Provaznik, Matej Santi, Cornelia Szabó-Knotik

Musikgeschichte anders erzählen? Das Beispiel der 1970er in Österreich.

Musikhistoriographie in der Zeit der Digitalisierung

\section{Mauro Fosco Bertola}

„Ein Laut so klagevoll”. Lohengrin zwischen Richard Wagner und Salvatore Sciarrino

\section{Matthieu Cailliez}

Europäische Rezeption der Berliner Hofoper und Hofkapelle von 1842 bis 1849

\section{lacopo Cividini}

Zwischen klassischer Musikphilologie und angewandter Informatik:

Die Digitale Mozart-Edition (DME) der Stiftung Mozarteum Salzburg

\section{Marko Deisinger}

Fortschrittliche Technologie im Dienste eines Antimodernisten.

Heinrich Schenker und der österreichische Rundfunk

\section{Norbert Dubowy}

Vom Kritischen Bericht zur Kritischen Dokumentation am Beispiel der Digital-interaktiven Mozart-Edition

\section{Markus Engelhardt}

Musik zwischen Nation Building und Internationalität. Italien um 1900

\section{Maryam Haiawi}

Das Oratorium im Spannungsfeld der Konfessionen: 


\section{Judith I. Haug}

"Manch eine*r liegt, morgens noch trunken, im Rosengarten" - Rekonstruktionen

osmanischer Musikgeschichte in Gesangstextsammlungen

\section{Renate Koch}

Marcel Prawy und das erste Broadway-Musical im Österreich der Nachkriegszeit

Susanne Kogler, Julia Mair, Juliane Oberegger, Johanna Trummer

Erich Marckhl - Musikausbildung in der Steiermark nach 1945.

Brüche und Kontinuitäten

\section{Marie-Anne Kohl}

Die weinende Jury. "Geschlechtslose" Tränen bei globalen Musik-Castingshows?

\section{Fabian Kolb}

Tanztheater und filmische Ästhetik. Cineastische Einflüsse und Gestaltungsweisen in den Kompositionen für die Ballets Suédois 1920-1925

\section{Christian Lehmann}

Tempobezeichnungen von Julius Stockhausen für Die schöne Müllerin:

Ein Quellenfund

\section{Martin Link}

Signum et gens - Zur Gendersemiotik in Clara und Robert Schumanns Liederzyklus Liebesfrühling

\section{Livio Marcaletti}

„Strafspiel" und satirische Stilmittel in musikdramatischen Gattungen des frühen 18. Jahrhunderts

\section{Tobias Marx, Martin Lissner}

Thüringer Musikszene - Jugendmusikredaktionen als außerschulische musikbezogene Bildungskontexte

\section{Maho Naito}

Die Parallelität der Entstehungsprozesse der ersten beiden Symphonien Gustav

Mahlers: Instrumentation, Revision und Dirigierpraxis

\section{Elisa Novara}

Eine Schumann-Werkstatt? Zur Übertragbarkeit der Methoden vom Projekt 
Theodora Oancea, Joachim Pollmann, Jonas Spieker

Kollaborateure - Involvierte - Profiteure. Erarbeitung eines Online-Lexikons zur

Musik in der NS-Zeit

\section{Kiron Patka}

„Ich wollte eigentlich Sängerin werden." Berufsselbstbilder von Tontechniker*innen im Radio

\section{Siegwart Reichwald}

Die Leiden der jungen Clara: Das Klaviertrio Opus 17 als Ausdruck einer Neu-

Romantikerin

\section{Elisa Ringendahl}

Lied versus Oper - Pole musikalischer Gattungen bei Oscar Bie

\section{Benedikt Schubert}

Struktur und Exegese. Über Eigentümlichkeiten in der Arie "Des Vaters Stimme ließ sich hören" (BWV 7/4)

Uwe Seifert, Sebastian Klaßmann, Timo Varelmann, Nils Dahmen

Computational Thinking in der Musikwissenschaft: Jupyter Notebook als Umgebung

für Lehre und Forschung

\section{Yusuke Takamatsu}

Synthese als Modus der Prozessualität bei Schubert:

Sein spezifisches Wiederholungsprinzip im langsamen Satz

\section{Daniel Tiemeyer}

Johann Nepomuk Hummels Sonate in fis-Moll Op. 81 - Studien zu Entstehungs-

hintergrund, Rezeption und formalerStruktur

\section{Andrea van der Smissen}

Musikalische Innovation im Umfeld der Moderne und historischen Avantgarde in Ungarn

\section{Tim Ziemer, Holger Schultheis}

Psychoakustische Sonifikation zur Navigation in bildgeführter Chirurgie

\section{Magdalena Zorn}

Musik mit dem Radio hören: Über den Begriff der musikalischen Aufführung 
Gabriele Buschmeier in memoriam 


\section{Vorwort}

Die vorliegenden Bände dokumentieren die Jahrestagung der Gesellschaft für Musikforschung 2019. In den dreieinhalb Tagen vom 23. bis zum 26. September 2019 wurden in Paderborn und Detmold nicht weniger als 185 Beiträge präsentiert, verteilt auf diverse Symposien, Round tables, Freie Sektionen und Postersessions. Sie alle auf einen Nenner bringen zu wollen, ist ein Ding der Unmöglichkeit - und das ist gut so, ist es doch Ziel der Jahrestagungen, die große Vielfalt der Themen und Methoden des Faches Musikwissenschaft abzubilden. Um die thematische Vielfalt der freien Referate angemessen abbilden zu können und gleichzeitig den inhaltlichen Schwerpunkten der beiden hier publizierten Hauptsymposien ausreichend Raum bieten zu können, erscheinen diese in drei Bänden.

„Musikwissenschaft: Aktuelle Perspektiven": Der Titel der kleinen Reihe ist keine Verlegenheitslösung. Musikwissenschaft im Kontext der Digital Humanities; Musikwissenschaft und Feminismus; Musik und Medien; Musikalische Interpretation - schon die Felder, die von den vier Hauptsymposien bespielt wurden, wären noch vor wenigen Jahrzehnten allenfalls an der Peripherie das Faches zu finden gewesen. Sie entsprechen Arbeitsschwerpunkten der Lehrenden am Musikwissenschaftlichen Seminar der Universität Paderborn und der Hochschule für Musik Detmold, das die Tagung ausrichtete. Zugleich nehmen sie Bezug auf aktuelle Ereignisse und Entwicklungen. So erwuchs das von Andreas Münzmay und Joachim Veit organisierte Symposium „Brückenschläge - Informatik und Musikwissenschaft im Dialog" unmittelbar aus den Erfahrungen im Virtuellen Forschungsverbund Edirom (ViFE) und im fakultäten- und hochschulübergreifenden Zentrum Musik-Edition-Medien (ZenMEM). Der 200. Geburtstag von Clara Wieck/Schumann war der Anlass für das von Rebecca Grotjahn geleitete Symposium „Die Begleiterin - Clara Schumann, Lied und Liedinterpretation", das in enger Kooperation mit der Hochschule für Musik Detmold durchgeführt wurde. Das Hauptsymposium „Brückenschläge" wird in einem separaten Band publiziert (Bd. 3 der vorliegenden Reihe). Im Rahmen dieses Symposiums führte die von Stefanie Acquavella-Rauch geleitete Fachgruppe Digitale Musikwissenschaft eine Posterpräsentation durch, die von den Beiträger*innen erfreulicherweise zu kürzeren Texten umgearbeitet wurden, sodass sie hier ebenfalls, zusammen mit den Postern,

publiziert werden können. Hinzu kommen einige Beiträge, die bereits bei der Jahrestagung 2018 in Osnabrück präsentiert wurden. Auch das Hauptsymposium "Die Begleiterin" wird in einem eigenen Band (Bd. 2) publiziert. Die Beiträge zu den beiden anderen Hauptsymposien hingegen werden an anderen Orten veröffentlicht; in Band 1 („Freie Beiträge zur Jahrestagung der Gesellschaft für Musikforschung 2019") der vorliegenden Publikation finden sich jedoch Einführungen und Abstracts. Das Symposium „Komponieren für das Radio" unter Leitung von Antje Tumat und Camilla Bork (Katholieke Universiteit Leuven) behandelte Einflüsse des Mediums auf Kompositionsprozesse sowie durch radiophone Kompositionen bzw. radiophonen Klang ausgelöste Diskurse. Sarah Schauberger und Cornelia Bartsch (Universität Oldenburg) nahmen das 25-jährige Jubiläum der Fachgruppe Frauen- und Genderstudien zum Anlass für einen Generationenaustausch zum Thema "Musikwissenschaft - Feminismus - Kritik": Was wa- 
ren vor einem Vierteljahrhundert Inhalte und Aufgaben einer feministischen Musikwissenschaft und wie kann sich diese heute positionieren?

Bewusst haben wir im Tagungsbericht auf inhaltliche Eingriffe in die Beiträge verzichtet. ${ }^{1}$ Das gilt besonders für die Freien Referate: Es galt, den Charakter der Jahrestagung als Forum für ,freie', d. h. innovative und auch experimentelle Gedanken zu wahren. Einige Kolleg*innen, die die Tagung mit Vorträgen und Posterpräsentationen bereichert hatten, haben sich gegen eine Publikation im vorliegenden Band entschieden - sei es, weil sie eine Möglichkeit fanden, ihre Beiträge in einem inhaltlich passenderen Rahmen zu veröffentlichen, sei es, weil ihre Überlegungen in ihre entstehenden Qualifikationsschriften fließen sollen, oder sei es, weil sie von den Autor*innen in der vorgetragenen Form zunächst verworfen wurden. Auch damit erfüllt eine Freie-Referate-Sektion ihren Zweck: Die Diskussionen mit der versammelten Fach-Öffentlichkeit sollen dabei helfen, Gedanken weiterzuentwickeln und zu verändern. In diesem Sinne sei allen Beteiligten - den Autor*innen, den nichtpublizierenden Referent*innen und den Mit-Diskutant*innen - ganz herzlich gedankt für ihr Mitwirken bei der Tagung.

Unser herzlicher Dank gilt einer Reihe weiterer Personen, die zum Gelingen dieser drei Bände beigetragen haben. Hier ist besonders Jonas Spieker zu nennen, der uns tatkräftig bei der Redaktion geholfen hat. Andrea Hammes (SLUB Dresden) sei herzlich für die Aufnahme unseres Bandes auf musiconn.publish gedankt - wir freuen uns, damit unsererseits zur Etablierung dieser innovativen Publikationsplattform beizutragen.

Erneut möchten wir an dieser Stelle allen Menschen danken, die uns bei der Organisation, Ausrichtung und Finanzierung der Tagung selbst unterstützt haben: der Präsidentin der Universität Paderborn, Prof. Dr. Birgitt Riegraf, dem Rektor der Hochschule für Musik Detmold, Prof. Dr. Thomas Grosse, den Kolleginnen und Kollegen der beiden beteiligten Hochschulen, dem Vorstand der Gesellschaft für Musikforschung, der Universitätsgesellschaft Paderborn und allen Sponsoren. Besonders dankbar sind wir den Mitarbeiter*innen und den studentischen bzw. wissenschaftlichen Hilfskräften des Musikwissenschaftlichen Seminars, die bei der Vorbereitung und Ausrichtung der Tagung immensen Einsatz zeigten - stellvertretend sei an dieser Stelle Johanna Imm erwähnt, die zusammen mit Nina Jaeschke das Herz des Organisationsteams bildete.

Wir widmen diese Reihe Dr. Gabriele Buschmeier, dem langjährigen Vorstandsmitglied der Gesellschaft für Musikforschung, die kurz vor der Publikation dieses Bandes unerwartet verstarb.

Detmold, im September 2020

Rebecca Grotjahn und Nina Jaeschke

Zitation: Rebecca Grotjahn und Nina Jaeschke, „Vorwort”, in: Freie Beiträge zur Jahrestagung der Gesellschaft für Musikforschung 2019, hrsg. von Nina Jaeschke und Rebecca Grotjahn (= Musikwissenschaft: Aktuelle Perspektiven. Bericht über die Jahrestagung der Gesellschaft für Musikforschung 2019 in Paderborn und Detmold, Bd. 1), Detmold 2020, S. IX-X, DOI: 10.25366/2020.43.

1 Freigestellt war den Autor*innen auch, ob sie sich für eine gendersensible Sprache entscheiden bzw. welche Form des Genderns sie bevorzugen. 